ORIGINAL ARTICLE

\title{
Anatomy of a patient safety event: a pediatric patient safety taxonomy
}

\author{
D M Woods, J Johnson, J L Holl, M Mehra, E J Thomas, E S Ogata, C Lannon
}

Qual Saf Health Care 2005;14:422-427. doi: 10.1136/qshc.2004.013573

See end of article for authors' affiliations

.....................

Correspondence to: Dr D M Woods,

Northwestern University, Weibildt Hall, Chicago, IL 60611, USA; woods@ northwestern.edu

Accepted for publication 19 August 2005

\begin{abstract}
Background: Idiosyncratic terminology and frameworks in the study of patient safety have been tolerated but are increasingly problematic. Agreement on standard language and frameworks is needed for optimal improvement and dissemination of knowledge about patient safety.

Methods: Patient safety events were assessed using critical incident analysis, a method used to classify risks that has been more recently applied to medicine. Clinician interviews and clinician reports to a web based reporting system were used for analysis of hospital based and ambulatory care events, respectively. Events were classified independently by three investigators.

Results: A pediatric patient safety taxonomy, relevant to both hospital based and ambulatory pediatric care, was developed from the analysis of 122 hospital based and 144 ambulatory care events. It is composed of four main categories: (1) problem type; (2) domain of medicine; (3) contributing factors in the patient (child-specific), environment (latent conditions) and care providers (human factors); and (4) outcome or result of the event and level of harm. A classification of preventive mechanisms was also developed. Inter-rater reliability of classifications ranged from $72 \%$ to $86 \%$ for sub-categories of the taxonomy.

Conclusions: This patient safety taxonomy reflects the nature of events that occur in both pediatric hospital based and ambulatory care settings. It is flexible in its construction, permits analysis to begin at any point, and depicts the relationships and interactions of elements of an event.
\end{abstract}

S ince the 1999 release of the IOM report "To Err Is Human", ${ }^{\prime}$ patient safety has become a distinct and proliferating field of study. Idiosyncratic terminology and frameworks for the study of medical errors and related injuries have been tolerated but are an increasing problem. The creation of, and agreement on, standard language and conceptual frameworks are needed for optimal discussion, improvement, and dissemination of knowledge on patient safety. A patient safety taxonomy that describes the types of events that occur, distinguishes the relevant contributory factors in the process and context of their occurrence, and builds a framework for understanding the relationships among the components of these events is needed. The Institute of Medicine has articulated this need in the recent report "Patient Safety: Achieving a New Standard of Care" in which it recommends that terminology should be standardized. $^{2}$

To address these needs and to ensure that the context of pediatric care is understood and included in any standardized structure and terminology, we developed a pediatric patient safety taxonomy derived from critical patient safety events reported in pediatric inpatient and ambulatory care settings. The events were classified in terms of:

- what happened;

- in what context;

- what contributed to the event (such as patient specific factors, human factors, organizational and systemic factors); and

- what occurred as a result of the event (for example, medical outcome, level of harm).

The purpose of any taxonomy is to organize a large body of information for a particular function and to provide a framework to answer a particular set of questions. A good taxonomy provides a meaningful structure for the information and group specific features of information into categories that describe, explain, and enable predictions. For example, in the periodic table of elements the boundaries of elements are described, the number of protons, electrons, and molecular weight is enumerated and explained, and the particular placement of an element on the periodic table predicts the nature of the element (for example, whether it is a metal or a salt). In many existing classification systems of medical errors and safety event types, the category levels are neither parallel nor exclusive. ${ }^{3}$ Outcomes such as falls are mixed in with medical processes such as administration of the wrong medication.

The categories of a taxonomy are generally assessed according to the following criteria:

- ability to answer the intended question;

- parallelism of the categories or equivalency of the categories within each level of the taxonomy;

- mutual exclusivity of categories; and

- classification of all incidences, given sufficient information, into an existing category.

A taxonomy can continue to evolve as new manifestations are found, such as in the taxonomy of genus and species.

The primary purpose of this patient safety taxonomy is to organize the available and developing knowledge about patient safety for use in research and clinical practice. The taxonomy provides a framework to describe the occurrence rates of specific types of events, the relationships between the contributing factors, and the domains of medicine in which events occur. It also offers a classification of event outcomes that can help to identify interventions that should predictably reduce patient harm and lead to patient safety improvement.

To date, no other studies have sought to develop a classification based on the review of events or contexts of events in pediatric medical care. There is also evidence that 
children and adults experience different types and frequencies of risk for patient safety problems. ${ }^{4}$ In adults, surgical errors $(48 \%)$ and medication errors $(19 \%)$ are the most frequent types of error, ${ }^{5}$ whereas events in the context of birth (a context that does not exist for adults) and diagnostics are most common in children. ${ }^{4}$ We therefore chose not to adapt a taxonomy derived from an adult based medical context but, rather, to develop a new taxonomy of pediatric events. We further evaluated the extent to which the classification categories are similar or differ from other existing classifications.

\section{METHODS}

\section{Critical incident analysis}

A critical incident analysis was performed to understand better the types of problems that occur in children's medical care and to categorize these events, the domain of medicine in which these events occurred, and to describe the contributory factors involved. Critical incident analysis is a qualitative observational technique that uses inductive methods to understand a phenomenon better. ${ }^{6}$ This method was established initially in aviation in the 1950 s to provide a better understanding of aviation training accidents and has become an accepted method of studying patient safety in medicine. It is not concerned with duplication of events. A single event with a particular pattern is sufficient to identify the existence of this pattern in the context of medicine and for the process of classification. This method is primarily used to document and understand the types of patterns that exist-for example, in the description and enumeration of species in biology where, if by the criteria you see only one organism in the species, the species exists.

Data collection in a critical incident analysis study consists of collecting factual reports from an individual's own observation of their behavior or of the behavior of others. ${ }^{6}$ In this study, reports about an event were collected from interviews of hospital based pediatric clinicians who recounted their observations of themselves or of others, and through a web based reporting system used by ambulatory care pediatricians.

\section{Hospital based data collection}

Hospital based pediatric clinicians (attending physicians, residents, nurses, and pharmacists) from an urban children's hospital were asked to participate in in-person audiotaped interviews to describe clinical situations "in which something did not go quite right or did not go as planned in the medical care of a child". This question was based on the definition provided in the IOM report "To Err Is Human: Building Safer Health Care" and was transformed to encourage reporting into a conversational construct. ${ }^{1}$ The question was designed to elicit events which resulted in harm, those in which no harm resulted, or events that would have resulted in harm had it not been for the intervention of another healthcare professional. We randomly chose subjects from the personnel lists of hospital and medical school departments to include different units (medical, surgical, pediatric intensive care unit (PICU), emergency department (ED)) and specialties (such as neurology, surgery, and cardiology). Selected subjects were mailed a letter inviting them to participate and were then called to schedule an interview. A standardized interview protocol was used to identify patient safety situations in which the clinicians had participated or had observed within the previous 6 months. Clinicians were also asked to recommend potential preventive mechanisms.

\section{Ambulatory care data collection}

A separate critical incident analysis was performed for data gathered in the ambulatory care setting. Pediatric physicians reported errors using a web based reporting tool as part of a project funded by the Agency for Healthcare Quality (AHRQ) entitled "Learning from Errors in Ambulatory Pediatrics (LEAP)". "The reporting tool, much like the interview protocol used for hospital based data collection, included several open ended questions designed to elicit "what happened" and the sequence of events. There were also several detailed questions about the patient and how the event was discovered. The tool was developed as an internet based secure (encrypted/digital certificate) tool to ensure the confidentiality of the reporter and of the reporting practice. Data collection was completed in collaboration with the Pediatric Research in Office Settings (PROS) network which is a practice based research network established by the American Academy of Pediatrics in 1986, made up of 553 practices ( 1609 practitioners) providing care to over 2 million children annually. Five PROS practices participated in the pilot study of the web based reporting tool. Following the pilot study, additional sites were recruited. Sites were selected to ensure a geographically, ethnically, and socioeconomically diverse population. A total of 14 sites participated.

\section{Analysis of data}

Text based constant comparative analysis was performed on the transcribed interview data and on the web based reports. Categories of problem type, the domain of medicine in which the patient safety event occurred, factors contributing to the occurrence of the event, the resulting outcomes, and potential preventive mechanisms were all inductively developed through application of the constant comparative method. ${ }^{8}$ This process included the following iterative steps:

- overall review of the textual data;

- detailed review of a few text reports to formulate meaning;

- review of additional reports to develop preliminary categories;

- coding of data by category and determination of the need for new categories and grouping of related categories to develop overarching categories;

- sorting of the data by category and performance of preliminary analysis of each category;

- deductive review for parallelism and clarity of categories; and

- classification of all of the data into the developed categories. ${ }^{3}$

The final step was performed using a triangulated transcript based approach involving three independent reviewers (two pediatricians and one patient safety researcher). Discrepancies in coding were resolved by consensus. Reliability of the initial level of agreement of the classifications was assessed.

This patient safety taxonomy reflects the nature of events that occur in both pediatric hospital based and ambulatory care settings and was tested for reliability, validity, mutual exclusivity, and parallelism of the categories.

\section{RESULTS}

Thirty five pediatric clinicians from different units and specialties described 167 independent patient safety events. No selected participant refused to participate. Office based pediatric physicians reported an additional 147 independent events to the web based LEAP reporting system.

The reliability of the classifications ranged from $72 \%$ to $86 \%$ agreement among the reviewers. To assess the ease of classification and applicability to a broad range of events, 11 clinicians who had received no training with this patient 


\section{Table 1 Pediatric Patient Safety Taxonomy}

(I) Event type

(1) Problematic decision

(2) Problematic execution

(3) Problematic communication

(4) Technical/mechanical malfunction

(5) Insufficient information

(II) Domains of medical care

(1) Preventive medicine

(A) Immunization

(B) Preventive screening

(2) Diagnostics

(A) Medical history and physical examination

(B) Tests

(C) Reading, recording, and interpreting results

\section{(3) Treatment}

(A) Medications, blood products, fluids, diet

(a) Ordering

(b) Transcribing

(c) Dispensing

(d) Administration

(B) Surgical and non-surgical procedures

(a) Preparation

(b) Procedure

(C) Appointment scheduling, referral, and follow up communications

(D) Other medical treatments

(a) Psychiatric

(b) Social services

(c) Discharge planning

(4) Patient monitoring

(5) Patient communication

(A) Preventive care

(B) Diagnostic

(C) Medication

(D) Surgical care

(E) Post-surgical care

(F) Other medical treatment

(6) Patient identification

(7) Equipment

(A) Equipment malfunction

(B) Equipment availability

(C) Use of equipment

(8) Administrative

(A) Medical record related

(B) Other clinically significant administrative

(III) Contributing factors

(IIIA) Patient/child specific factors

(1) Physical characteristics

(A) Small size

(B) Morphology

(C) Varied size and morphology

(2) Development

(A) Physiological

(a) Underdeveloped systems

(b) Epidemiology

(c) Growth

(d) Child specific signs and symptoms

(B) Cognitive, social-emotiona

(a) Ability to communicate

(b) Ability to understand

(c) Ability to control/regulate behavior

(3) Minor legal status

(A) Medical management

(B) Decision making and consent

(C) Confidentiality

(D) Supervision

(4) No contributing patient/child specific factor

(5) Insufficient information to determine

(IIIB) Human factors

(1) Cognitive

(A) Knowledge

(B) Confusion

(C) Assumptions

(D) Inattention

(2) Physical

(A) Fatigue

(B) Physical pain

(C) Physical performance of a task

(3) Emotional

(A) Fear

(B) Dislike

(C) Emotional stress

(4) None

(5) Unclassifiable/insufficient information

\section{Table 1 Continued}

(IIIC) Latent conditions

(1) Organizational systems

(A) Systems for clinical communication

(a) Across departments

(b) Across professions

(B) Systems of medication provision

(a) Ordering

(b) Preparing

(c) Administration

(C) Infection control systems

(a) Hygiene

(b) Prophylactic antibiotics

(c) Infected clinicians/visitors

(d) Evaluation

(D) Systems of training

(E) Systems for equipment acquisition and maintenance

(2) Culture

(3) Structure/framework of medicine

(4) Physical environment design

(5) Staffing

(IV) Outcomes

(IVA) The result of the problem

(1) Near miss/good catch

(2) Adverse drug event

(3) Nosocomial infection

(4) Cardiac arrest

(5) Respiratory failure

(6) Wrong treatment/procedure

(7) Treatment of wrong patient

(8) Wrong site surgery

(9) Laceration

(10) Excess bleeding

(11) Loss of sensory input

(12) Paralysis

(13) Other neurological impairment

(14) Organ system impairment

(15) Fall

(16) Burn

(17) Fracture

(18) Failed procedure

(19) Breech of confidentiality

(20) Adverse blood transfusion reaction

(21) Patient missing

(22) Unnecessary protective services involvement

(23) Delayed growth/development

(24) Disorientation

(25) Loss of consciousness

(26) Return to the ICU within 48 hours

(27) Pain

(28) Social disruption

(29) None of the above but resulted in hospital

(30) Admission or extended hospital stay

(31) Death

(32) Insufficient information to determine

(IVB) Level of harm

(1) Near miss, error did not reach the patients, no harm

(2) Reached the patient, no harm

(3) Emotional harm

(4) Minor temporary harm

(5) Major temporary harm

(6) Minor permanent harm

(7) Major permanent harm

(8) Death

safety taxonomy were asked to classify 164 incident reports into the taxonomy categories (table 1).

\section{Taxonomy for pediatric patient safety problems}

Figure 1 provides the framework for the pediatric patient safety taxonomy and depicts the relationships and interactions of the components of the taxonomy. Table 1 shows that the pediatric patient safety taxonomy is composed of four main categories:

- problem type;

- domain of medical care in which the patient safety event occurred: 


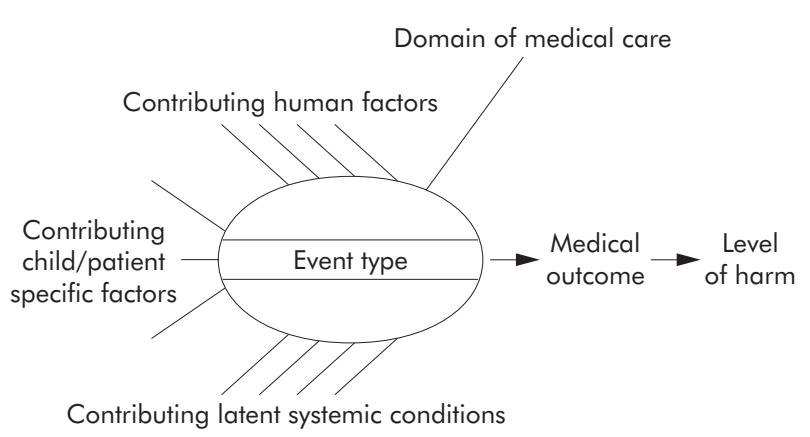

Figure 1 Conceptual model of a patient safety taxonomy.

- contributing factors in the patient (child specific), environment (latent conditions), care providers (human factors); and

- outcome (the result of the event and level of harm).

The resulting patient safety taxonomy is flexible in its construction and permits a classification or an analysis to begin at any point, depending on the purpose of the analysis. For example, analysis can begin with the question of identifying the domain of medical care that leads to the greatest level of harm, or the most frequent domain of medical care in which a particular event type occurs, or the factors that contribute most commonly to a particular problem type or a particular domain of medical care. This classification system also enables comparison of near miss events with adverse events.

As part of the critical incident analysis, clinicians were asked to recommend methods to prevent the events they had described. From these recommendations a set of classifications of preventive mechanisms was developed called "PERILS"-Policy, Education and training, Resources, Improving Communication, Legal, and Study (table 2).

The taxonomy provides common descriptors, permits an understanding of the common features and dynamics of a patient safety event, and provides a tool for patient safety analyses. Analysis based on this system of classification can also direct future patient safety prevention activity and organizational learning through the identification and prediction of the complex relationships in a patient safety event, and links them to methods of prevention and strategies for intervention.

\begin{tabular}{l} 
Table 2 Taxonomy of patient safety preventive \\
mechanisms \\
\hline Patient safety preventive mechanisms ('PERILS") \\
\hline (1) Policy \\
(A) Compliance with existing policy \\
(B) Addition or change of policy \\
(2) Education and training \\
(3) Resources \\
(A) Staffing \\
(B) Technology and smart design \\
(C) Equipment \\
(D) Space \\
(E) Time \\
(4) Improving communication \\
(A) Across departments \\
(B) Across professions \\
(C) Between clinicians and patients and families \\
(5) Legal action \\
(6) Study, monitor, analyse problematic situations \\
\hline
\end{tabular}

\section{DISCUSSION}

This pediatric patient safety taxonomy is based on data obtained from both individual interviews with hospital based pediatric clinicians and web based reports in ambulatory pediatrics. The categories in this taxonomy have been validated for both of these medical care contexts. Application of this taxonomy to specific cases is shown in table 3.

To date, several patient safety taxonomies have been developed and are currently being used. However, each taxonomy was developed using a different methodology, with a slightly different focus, and was validated for different medical care settings. The Applied Strategies for Improving Patient Safety (ASIPS), a voluntary patient safety reporting system, used a modified malpractice insurance claims risk assessment structure to model a medical error taxonomy and to collect events, ${ }^{9}$ and was validated in an ambulatory care practice based research network. The Australian Incident Monitoring Study (AIMS) was developed to be a "generic occurrence classification" to categorize incident reports from all types of providers ${ }^{10}$ based on natural language mapping of terms into a hierarchical tree. The Medical Error Reporting System-Transfusion Medicine (MERS-TM) is based on the Einhoven model, a safety reporting taxonomy used in other high risk industries and developed by an interdisciplinary team, ${ }^{11}{ }^{12}$ and was validated for collection of transfusion related events. It was then expanded and validated for the adult inpatient setting. The Joint Commission for Accreditation for Healthcare Organization (JCAHO) Patient Safety Event Taxonomy was developed through a literature review of potentially relevant existing taxonomies, included multiple classifications, and was then tested by application to hospital based sentinel events submitted to the $\mathrm{JCAHO}^{13}$ events that generally result in higher levels of related harm. The JCAHO taxonomy is not, however, validated to distinguish and describe events which were prevented before they reached the patient or resulted in minimal harm. The Linnaeus Primary Care Collaborative, International Taxonomy of Medical Errors in Primary Care is a primary care based patient safety taxonomy that used natural language mapping specifically designed for events that occur in the ambulatory care setting. ${ }^{2}$ This taxonomy is capable of describing events that did or did not result in harm and includes near miss events that did not reach the patient. However, this taxonomy was not designed or validated for events that occur in the inpatient hospital based setting. The Cognitive Taxonomy proposed by Jhang et al ${ }^{14}$ focuses on the human factor of cognition as a central aspect of error in medical contexts and illuminates the contribution and link between features of cognition to safe design and safety improvement strategies. This taxonomy goes beyond providing frequencies of events and permits the development and assessment of potential improvement strategies by classifying human factor causes and by providing finely granulated levels of categorization.

In comparison with these other systems of classification, the pediatric patient safety taxonomy described here provides an integrated hospital based and ambulatory care patient safety classification system and includes categories not previously described in other taxonomies. The contexts and processes of ambulatory care differ in many ways from those in hospital based care, ${ }^{15}$ and the nature of patient safety events in this setting can be more meaningfully described with the addition of new and distinct categories. For example, much of the focus in primary care is dedicated to preventive medicine. While many of these activities could be subsumed under a "diagnostics" category, to do so risks losing the unique significance of these activities and missing the particular ways that error and injury occur in ambulatory 
Table 3 Application of the taxonomy

Case 1

"Yesterday I saw a girl who had been followed for five years by a gastroenterologist for constipation. In the last year and a half it had been noted that she had not gained any weight at all ... The gastroenterologist did a lot of tests, very focused on looking to exclude the diagnosis of inflammatory bowel. I saw her yesterday, did some simple tests, and it became quite clear that her kidneys were not working at all as they should be. A very simple urine test would have picked this up months ago."

Domain of medicine

Problem type

Diagnostics

Contributing factors

Patient/child specific factors

Human factors

Latent conditions

Human action: problematic decision

Outcome

Outcome

Level of harm

Physiological development

Cognitive

Structure

Case 2

"Patient [was] prescribed a medication that the patient was known to be allergic [to] ... [the patient has a] past history of rash. The prescription was filled but the [allergy] was noted prior to giving the medication to the patient."

Domain of medicine

Problem type

Contributing factors

Patient/child specific factors

Human factors

Latent conditions

Delayed growth

Major temporary

Outcome

Outcome

Level of harm

Medication ordering: wrong medication

Human action: problematic decision

None

Physical

Medication systems

Near miss

No harm, good catch

Case 3

"We had a medication error ... it was a new nurse. We pretty much have a flat out chart and it's pretty selfexplanatory. It's fantastic stuff, like what to do, how much is in there, what to run it at-it's an excellent chart. And any [patient] over $30 \mathrm{~kg}$ gets $1 \mathrm{cc}$ of Isuprel in a $50 \mathrm{cc}$ bag. So less than $30 \mathrm{~kg}$, it is all charted out. So it was a less than $30 \mathrm{~kg}$ baby, but she was out of the habit because the majority of our kids that we do with SVT are greater than $30 \mathrm{~kg}$... So when we had someone less than $30[\mathrm{~kg}]$, she knew to go to the chart, but out of pure habit, she just put the cc in there and the kid had extra Isuprel."

Domain of medicine

Problem type

Contributing factors

Child specific factors

Human factors

Latent conditions

Medication administration: wrong concentration

Human action: problematic execution

Outcome:

Medical outcome

Variable size and morphology

Cognitive

Medication systems

Level of harm

$3 \times$ overdose

Minor temporary harm

Case 4

"... A case of a child with a large ... [abdominal] tumor, a vessel arising apparently from the chamber that appeared to be an ... artery, appeared to flow right into the tumor directly and it was pulsating and it was a vessel that appeared to be the [artery we were trying to ligate] ... we had come around to the medial side and got control of the artery and the vein ... to ligate them and take them out ... that would allow those vessels to be divided which will allow the [organ] to be elevated... The vessel was identified. The senior pediatric surgical fellow was my assistant... very experienced for this procedure ... We both tracked this vessel out, started going to the [organ], tracked it back to the point of origin, and we were convinced that it was the artery we thought it was. We ligated it, we immobilized the [organ] ... So this vessel that we thought was arising from the aorta ... was actually the superior mesenteric artery ... so the artery was divided. Upon release of the [organ] and of the mass, it was apparent that the orientation of the aorta and this vessel was not what I thought it was."

Domain of medicine Procedure

Problem type Human action: problematic execution

Contributing factors

Child specific factors None

Human factors Cognitive

Latent conditions Systems

Outcome

Medical outcome Cut wrong vessel

Level of harm Major temporary

care. ${ }^{16}$ Preventive medicine involves following a protocol of screening, usually when there may be no symptoms present, in contrast to determining the causes of a set of symptoms. Other important differences related to the ambulatory setting include error prone activities such as appointment scheduling, referrals to other providers, follow up and communication of test results, and refilling prescriptions. In this taxonomy, categories were assigned for these important ambulatory care functions. In addition, the administrative functions of office based ambulatory care have been shown in this and other studies ${ }^{3}$ to be important categories for which errors and related injuries are common. Furthermore, our taxonomy integrates the hospital based and ambulatory care contexts into one comprehensive classification. With the exception of the AIMS, none of the existing taxonomies was designed and validated to collect and describe events that occur in both the inpatient and ambulatory care settings.

This pediatric patient safety taxonomy was developed in the context of pediatric health care. To the extent that categories and definitions are similar to those developed in 
adult medicine, this study validates their applicability for pediatrics. However, the entire history of pediatrics has been a process of adapting medical processes for the fundamental physiological, biochemical, and physical differences between children and adults. This taxonomy incorporates the characteristics of children that contribute to patient safety risk and must therefore be taken into consideration to improve the safety of children's medical care. ${ }^{4}$ None of the existing taxonomies includes factors related to child specific contributory risks, although these factors have been shown to contribute to the occurrence of medical errors and related injuries. ${ }^{4}$

Additional strengths of this taxonomy include parallelism of the levels of categories, mutual exclusivity of the categories, flexibility of the taxonomy structure, descriptive and predictive ability of the classification, and content not included in other systems of classification such as contributing patient specific risk factors. The taxonomy has been designed and structured to be both descriptive and predictive and has the ability to display both the relationships between various components and the pathways from the contributing factors to the outcome.

This taxonomy provides a conceptual model for a higher order classification of error-that is, event type, domain of medicine, contributing human factors, contributing patient specific factors, contributing latent systemic conditions, medical outcome, level of harm-that reflects the relationships of these categories and depicts the path of error and injury occurrence. This organization of higher order classification categories into a conceptual model provides a path for future patient safety research.

The pediatric patient safety taxonomy responds to many of the requirements for classification and definition laid out in the IOM report outlined in "Achieving New Standards for Patient Safet $^{\prime \prime 2}$ to develop a framework for data that will facilitate the "discovery, analysis, understanding of, and learning from patient safety events". This report recommends a set of "well integrated, non-redundant terminologies" for clinical care, and suggests that the patient safety elements needed for classification include: what (the event type), when (the aspect of care or care process), why (the dominant causes), the severity of the event, preventability of the event, product information (drugs, devises, blood, etc), and patient information. ${ }^{2}$ This pediatric patient safety taxonomy includes many of the desired elements. Recommended categories such as when the event occurred and who was involved were not included in the taxonomy but can easily be added when relevant.

The National Quality Forum (NQF), an organization comprised of healthcare researchers, institutions, and consumers, is engaged in a "national consensus process" to develop a standard taxonomy for patient safety. The process includes academia, healthcare institutions, and the public for a discourse, examination, critique, and refinement of a preliminary taxonomy for all types of medical care. NQF has preliminarily chosen the JCAHO-PSET taxonomy which will be reviewed and enhanced to include features that are currently not part of the taxonomy, particularly categories relevant to pediatric care, ambulatory care, and low harm or near miss events.

While the taxonomy described here could be applied to event data collection systems, its primary purpose is theoretical-to map and model the critical elements of patient safety events. Classification of this taxonomy to events will enable an analysis that facilitates the development, application, and evaluation of preventive strategies and the aggregation of events along categorical dimensions that further facilitate knowledge building, illuminating causes and the development of potential safety interventions. In this manner, the added categories and conceptual model of this taxonomy could inform the NQF consensus process already underway by providing a theoretical framework as well as elements that may be lacking in the taxonomy going forward.

\section{ACKNOWLEDGEMENTS}

The authors thank Kevin B Weiss MD, MPH for his insightful mentoring and thoughtful direction; Jack Shonkoff MD for his mentoring, careful critique, and instruction in the application of logic to the process of taxonomy development; Stuart Altman PhD for his guidance and direction in the performance of this research; Uri Wilensky PhD, Daniel Edelson PhD, and Sharona Levy PhD for their consultation regarding science and computer science taxonomies and criteria; and Penelope Peterson and Peter Budetti for their material support of this work.

\section{Authors' affiliations}

D M Woods, Institute for Healthcare Studies, Feinberg School of Medicine, Northwestern University, Chicago, IL, USA

J Johnson, Department of Medicine, University of Chicago, Chicago, IL, USA

J L Holl, Institute for Healthcare Studies and Department of Pediatrics, Feinberg School of Medicine, Northwestern University and Children's Memorial Hospital, Chicago, IL, USA

M Mehra, Feinberg School of Medicine, Northwestern University, Chicago, IL, USA

E J Thomas, Department of Medicine, University of Texas Medicial School, Honston, TX, USA

E S Ogata, Children's Memorial Hospital, Chicago, IL, USA

C Lannon, Center for Children's Healthcare Improvement, Department of Pediatrics, School of Medicine, University of North Carolina at Chapel Hill, Chapel Hill, NC, USA

This research was supported in part by a seed grant from the Children's Memorial Institute for Evaluation and Research. Research for this paper was done while the first author was a National Research Service Award postdoctoral fellow at the Institute for Health Services Research and Policy Studies, at the Feinberg School of Medicine, Northwestern University under an institutional award from the Agency for Healthcare Research and Quality.

This research was performed under the approval of the Institutional Review Boards of Children's Memorial Hospital and the University of Chicago.

\section{REFERENCES}

1 Kohn LT, Corrigan JM, Donaldson M, eds. To err is human: building a safer health system. Washington, DC: National Academy of Sciences, 1999.

2 Aspden P, Corrigan J, Wolcott J, et al. Patient safety: achieving a safer standard of care. Washington, DC: National Academy of Sciences, 2004.

3 Dovey SM, Meyers DS, Phillips RL, et al. A preliminary taxonomy of medical errors in family practice. Qual Saf Health Care 2002;1 1:233-8.

4 Woods DM, Thomas EJ, Altman S, et al. Adverse events and preventable adverse events in children. Pediatrics 2005;151:155-60.

5 Thomas EJ, Studdert D, Burstin HR, et al. Incidence and types of adverse events and negligent care in Utah and Colorado. Med Care 2000;38:261-71

6 Flanagan J. The critical incident technique. Psychol Bull 1954;51:327-58.

7 Mohr JJ, Lannon CM, Thoma KA, et al. Learning from Errors in Ambulatory Pediatrics (LEAP). In: Advances in patient safety: from research to implementation 2005

8 Creswell JW. Research design: qualitative and quantitative approaches. Thousand Oaks: Sage Publications, 1994.

9 Fernald DH, Pace WD, Harris DM, et al. Event reporting to a primary care patient safety reporting system: a report from the ASIPS collaborative. Ann Fam Med 2004;2:327-32.

10 Runciman RB, Helps SC, Sexton EJ, et al. A classification for incidents and accidents in the health-care system. J Qual Clin Practice 1998;18:199-211.

11 Battles JB, Kaplan HS, Van der Schaff TW, et al. The attributes of medical reporting systems: experience with a prototype medical event reporting system for transfusion medicine. Arch Pathol Lab Med 1998;122:231-8.

12 Kaplan HS, Battles JB, Van der Schaff TW, et al. Identification and classification of the events in transfusion medicine. Transfusion 1998;38:1071-81.

13 Chang A. Joint Commission for the Accreditation of Health Care Organizations: patient safety event taxonomy. Presentation to the American Academy of Family Practice Conference on Patient Safety Taxonomies, Orlando, FL, October, 2004

14 Zhang J, Patel VL, Johnson TR, et al. A cognitive taxonomy of medical errors. $J$ Biomed Inform 2004;37:193-204.

15 Hammons T, Piland NF, Small SD, et al. Ambulatory patient safety: what we know and need to know. J Ambulatory Care Manage 2003;26:63-82.

16 Gaeta T. Screening and diagnostic tests. E-Medicine. http://emedicine.com/ emerg/topic770.htm (accessed 19 May 2005). 